Provided for non-commercial research and education use. Not for reproduction, distribution or commercial use.

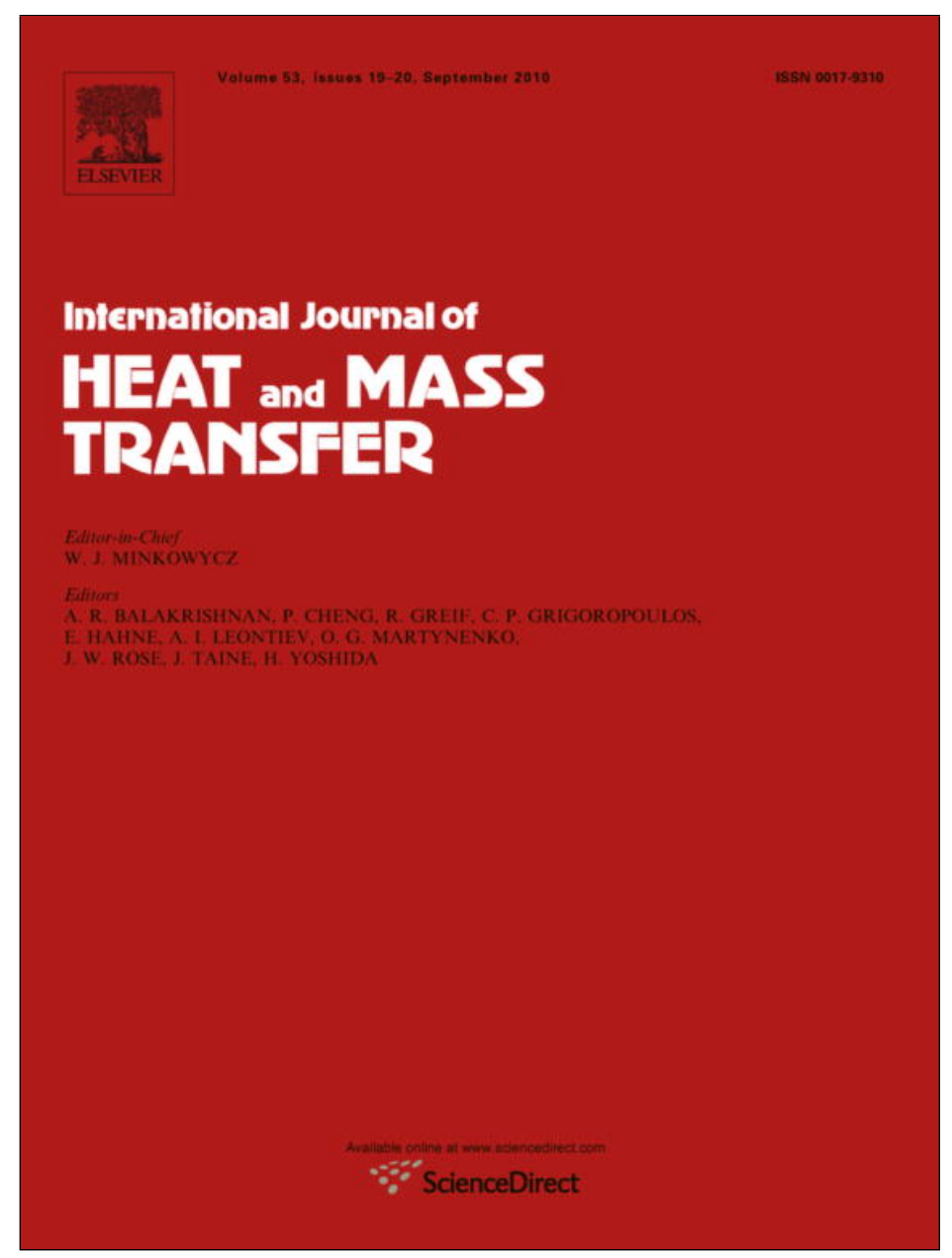

This article appeared in a journal published by Elsevier. The attached copy is furnished to the author for internal non-commercial research and education use, including for instruction at the authors institution and sharing with colleagues.

Other uses, including reproduction and distribution, or selling or licensing copies, or posting to personal, institutional or third party websites are prohibited.

In most cases authors are permitted to post their version of the article (e.g. in Word or Tex form) to their personal website or institutional repository. Authors requiring further information regarding Elsevier's archiving and manuscript policies are encouraged to visit:

http://www.elsevier.com/copyright 


\title{
Thermally induced two-phase oscillating flow inside a capillary tube
}

\author{
S.P. Das ${ }^{a}$, V.S. Nikolayev ${ }^{b, c, *}$, F. Lefevre ${ }^{a}$, B. Pottier ${ }^{b, c}$, S. Khandekar ${ }^{d}$, J. Bonjour ${ }^{a}$ \\ a Université de Lyon, CNRS, INSA-Lyon, CETHIL, UMR5008, F-69621 Villeurbanne Cedex, France \\ ${ }^{\mathrm{b}}$ ESEME, Service des Basses Températures, INAC, CEA-Grenoble, France \\ ${ }^{\mathrm{c}}$ ESEME, PMMH-ESPCI, 10, rue Vauquelin, 75231 Paris Cedex 5, France \\ ${ }^{\mathrm{d}}$ Department of Mechanical Engineering, Indian Institute of Technology Kanpur, Kanpur 208016, Uttar Pradesh, India
}

\section{A R T I C L E I N F O}

\section{Article history:}

Received 24 August 2009

Received in revised form 15 March 2010

Accepted 26 March 2010

Available online 31 May 2010

\section{Keywords:}

PHP

Two-phase fluid

Evaporation/condensation

Oscillation

Capillary meniscus

\begin{abstract}
A B S T R A C T
This paper deals with thermally induced meniscus oscillations in a two-phase system consisting of a liquid plug and a vapor bubble in a capillary tube of circular cross-section. This system represents the simplest version of a heat transfer device called "pulsating heat pipe" (PHP). Our purpose is to gain fundamental understanding of the physical processes that cause self-sustained thermally driven oscillations. A visualization experiment is performed and the oscillations of the liquid-vapor meniscus and the vapor pressure are observed. We propose next a theoretical model. It differs from existing models by the account of the two-phase equilibrium that occurs locally at the vapor-liquid interface and by introduction of the time varying wetting films through which the major part of the heat and mass transfer occurs. Results from the proposed model show a good agreement with the experiment.
\end{abstract}

(c) 2010 Elsevier Ltd. All rights reserved.

\section{Introduction}

The pulsating heat pipe (PHP) [1] is a long capillary tube bent into many turns and partially filled with a two-phase, usually single component, working fluid. The tube is simple, with no wick structure. When heated, the fluid spontaneously forms many vapor bubbles separated by liquid plugs inside the tube. Evaporation of liquid in the hot (evaporator) sections and subsequent condensation in the cold (condenser) sections creates oscillations of the bubble-plug structure. These oscillations are very important because they lead to a substantial increase of the heat transfer rate in comparison with other types of heat pipes [2]. In addition to the latent heat transfer characteristic for them, the sensible heat transfer occurs in PHP. While sweeping an evaporator section, a liquid plug accumulates the heat, which is then transferred to the condenser section when the plug penetrates there.

Because of their simplicity and high performance, PHPs are often considered as highly promising. Their industrial application is however limited because their functioning is not well controlled. Multiple parameters affect its thermal performance. During the last decade, researchers have extensively studied PHPs [3]. Tong et al. [4], Miyazki and Arikawa [5], Xu et al. [6] and Gi et al. [7] conducted flow visualization studies. Ethanol, methanol, deionized water and R142b were used in their studies. Their experiments

\footnotetext{
* Corresponding author at: ESEME, Service des Basses Températures, INAC, CEA-Grenoble, France. Tel.: +33 14079 5826; fax: +33 140795808 . E-mail address: vadim.nikolayev@espci.fr (V.S. Nikolayev).
}

confirmed the existence of self-sustained thermally driven oscillations in PHPs. Charoensawan et al. [8] and Yang et al. [9] performed experiments with different tube diameters, configurations, orientations and filling ratios and studied the thermal performance of PHPs in different conditions.

However, the functioning of PHPs is not completely understood. Unlike other types of heat pipes, the functioning of PHPs is nonstationary and thus difficult to model. A complicated interplay of different hydrodynamic and phase-exchange phenomena needs to be accounted for.

There are only few modelling approaches available in the literature. Zhang et al. [10], Dobson [11,12] studied the governing mechanism of the PHP using simple models. They studied a Ushaped miniature tube (i.e. single bend PHP) with a single liquid plug or a vapor bubble. The evaporation/condensation rate is assumed to be proportional to the difference of the temperatures of the vapor and the walls in contact with it. In the vapor bubble evolution equation this leads to terms analogous to those of sensible heat transfer between the vapor and the tube walls. The approach [10] has been extended by Shaffi et al. [13] to model both looped and unlooped PHPs with multiple vapor bubbles, liquid plugs and tube bends. This model has been used later by another team [14], also for multi-bubble PHP modelling.

It is well known from general considerations of thermal resistance that during the meniscus evaporation, an important contribution to the heat and mass transfer comes from thin liquid films that may cover the interior of the capillary. The local twophase equilibrium exists at the interface of microscopically thin 


\begin{tabular}{|c|c|c|c|}
\hline \multicolumn{4}{|c|}{ Nomenclature } \\
\hline$C_{\mathrm{f}}$ & friction coefficient & $\beta$ & coefficient in Eq. (15) \\
\hline$c_{\mathrm{vv}}$ & vapor specific heat at constant volume $(\mathrm{J} /(\mathrm{kg} \mathrm{K}))$ & $\delta$ & thickness (m) \\
\hline$D$ & vapor heat diffusivity $\left(\mathrm{m}^{2} / \mathrm{s}\right)$ & $\gamma$ & coefficient \\
\hline$d$ & tube diameter $(\mathrm{m})$ & $v$ & kinematic viscosity $\left(\mathrm{m}^{2} / \mathrm{s}\right)$ \\
\hline$F$ & force $(\mathrm{N})$ & $\rho$ & liquid density $\left(\mathrm{kg} / \mathrm{m}^{3}\right)$ \\
\hline$h_{\mathrm{lv}}$ & latent heat $(\mathrm{J} / \mathrm{kg})$ & $\tau$ & characteristic time scale $(\mathrm{s})$ \\
\hline$K$ & coefficient in Eq. (15) & & \\
\hline$k$ & heat conductivity $(\mathrm{W} /(\mathrm{m} \mathrm{K}))$ & \multicolumn{2}{|c|}{ Subscripts and superscripts } \\
\hline$L$ & length $(\mathrm{m})$ & $\mathrm{bl}$ & boundary layer \\
\hline$m$ & mass $(\mathrm{kg})$ & c & condenser, characteristic \\
\hline$P$ & oscillation period (s) & e & evaporator \\
\hline$p$ & pressure $(\mathrm{Pa})$ & eff & effective \\
\hline$q_{\text {sens }}$ & sensible heat flux $\left(\mathrm{W} / \mathrm{m}^{2}\right)$ & $\mathrm{f}$ & friction, film \\
\hline$R_{\mathrm{v}}$ & vapor gas constant $(\mathrm{J} /(\mathrm{kg} \mathrm{K}))$ & $\mathrm{i}$ & inertial \\
\hline $\operatorname{Re}$ & Reynolds number & 1 & liquid \\
\hline$S$ & tube section area $\left(\mathrm{m}^{2}\right)$ & $\mathrm{m}$ & meniscus \\
\hline$T$ & temperature $(\mathrm{K})$ & 0 & open end \\
\hline$t$ & time $(s)$ & $\mathrm{p}$ & pressure \\
\hline$U$ & heat transfer coefficient $\left(\mathrm{W} /\left(\mathrm{K} \mathrm{m}^{2}\right)\right)$ & $\mathrm{r}$ & reservoir \\
\hline$V$ & meniscus velocity $(\mathrm{m} / \mathrm{s})$ & sat & at saturation \\
\hline$x$ & meniscus position (m) & $\mathrm{t}$ & total \\
\hline$x_{\mathrm{f}}$ & film edge position (m) & $\mathrm{v}$ & vapor \\
\hline \multicolumn{4}{|c|}{ Greek symbols } \\
\hline$\alpha$ & coefficient in Eq. (15) & & \\
\hline
\end{tabular}

films [15] so that the interface is at saturation or very close to it. This effect was completely neglected in the above mentioned modelling approaches. Dobson $[11,12]$ has included a film in his singlebubble model. However the film mass exchange in his model was not related to the liquid-vapor equilibrium and the mass exchange was proportional to the difference of temperatures of the vapor and the wall, just like in the other works that did not treat the films at all. The single-bubble model of Zhang and Faghri [16] has taken a step forward by rigorously showing that most part of heat and mass exchange occurs via the films in the PHP. The shape of the curved meniscus including the film has been calculated.

Globally, the existing models describe oscillations of small amplitude. During these oscillations, the meniscus is located almost all the time in the condenser section. This contradicts most cited above experimental results where strong amplitude meniscus oscillations are observed. At each oscillation the meniscus sweeps both the condenser and evaporator. The objective of the present work is to propose a model that accounts for the twophase equilibrium at the vapor-liquid interface and can explain such large amplitude oscillations. Some experimental results will be presented and compared with the model.

\section{Experiments}

\subsection{Experimental setup}

The main part of the experimental setup (Fig. 1) is a capillary tube of $2 \mathrm{~mm}$ internal diameter. The evaporator section of the tube (of length $L_{\mathrm{e}}=15 \mathrm{~cm}$ ) is made inside an opaque copper cylindrical block. Three heating coils are wound around it. Power rating of each coil is $88 \mathrm{~W}$. The temperature of the evaporator is regulated to a constant value $T_{\mathrm{e}}$ with a tolerance of $\pm 1{ }^{\circ} \mathrm{C}$. The $25 \mathrm{~cm}$ long condenser section is made of transparent glass. It is enveloped by a transparent heat exchanger so that the coolant (silicon oil with low freezing temperature) flows around the condenser. Two ends of the exchanger are connected to a thermostatic bath (MINISTAT
CC with operating range $-25-150^{\circ} \mathrm{C}$ ). This bath operates with a maximum flow rate of $18 \mathrm{l} / \mathrm{min}$ at $600 \mathrm{mbar}$. The flow rate is $12 \mathrm{l} / \mathrm{min}$ at $300 \mathrm{mbar}$. It allows the condenser temperature $T_{\mathrm{c}}$ to be maintained constant within $\pm 0.1^{\circ} \mathrm{C}$. A small section of $1 \mathrm{~cm}$ between the condenser and the evaporator is insulated, and acts as the adiabatic section. Such a setup provides the fluid visualization only inside the condenser.

The closed left end of the evaporator section is connected to the KISTLER ${ }^{\circledR}$ piezoresistive absolute pressure sensor (type $4005 \mathrm{~B}$ and operating range of $0-20$ bar). The pressure sensor is calibrated in the pressure range $0-3$ bar with an accuracy of 2 mbar. A vacuum line, isolated from the main capillary tube through a shut-off valve, is also connected to the left evaporator end. This vacuum connection serves two purposes. First, it helps to remove any non-condensable gases present in the capillary tube before charging it with the working fluid. Second, it helps to control the position of the liquid-vapor meniscus in the beginning of the experiment.

The open right end of the capillary tube is connected to a large reservoir filled with the working fluid. A heating coil is wound around this reservoir to control the reservoir pressure. The entire system is first evacuated completely to remove any non-condensable gases. The liquid reservoir is then filled with the working fluid ( $n$-pentane), which is convenient because of its low saturation temperature at the ambient pressure. A pressure gauge and two thermocouples serve to measure the pressure and the temperature in the reservoir. The meniscus displacement $x$, counted from the left end of the evaporator, is measured from the condenser images acquired with a high speed (3000 frames/s) digital camera.

\subsection{Experimental results}

The oscillations in the system (i.e. its instability) appear when the difference between the temperatures $T_{\mathrm{e}}$ and $T_{\mathrm{c}}$ exceeds a threshold value. At small $T_{\mathrm{c}}$, the meniscus does not move out of the condenser (towards the fluid reservoir) which is convenient for the visualization; small $T_{\mathrm{c}}$ values $\left(0-10^{\circ} \mathrm{C}\right)$ are used. Fig. $2 \mathrm{a}$ 


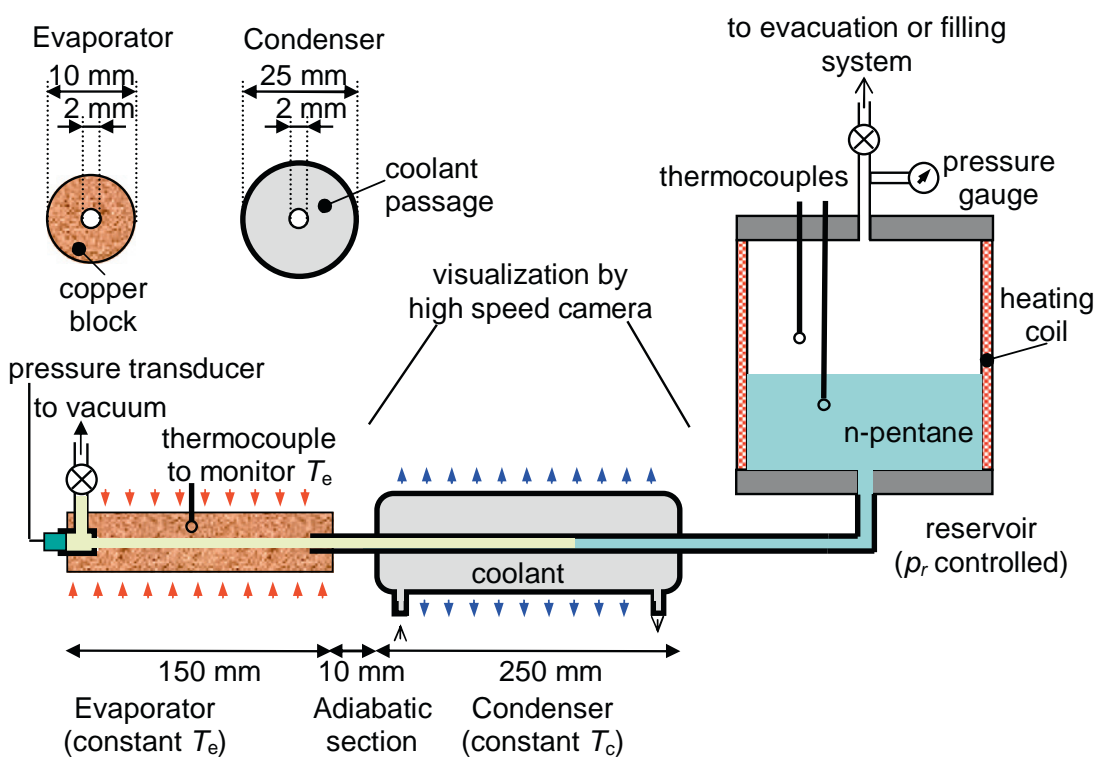

Fig. 1. Schematic of the experimental setup (not to scale).
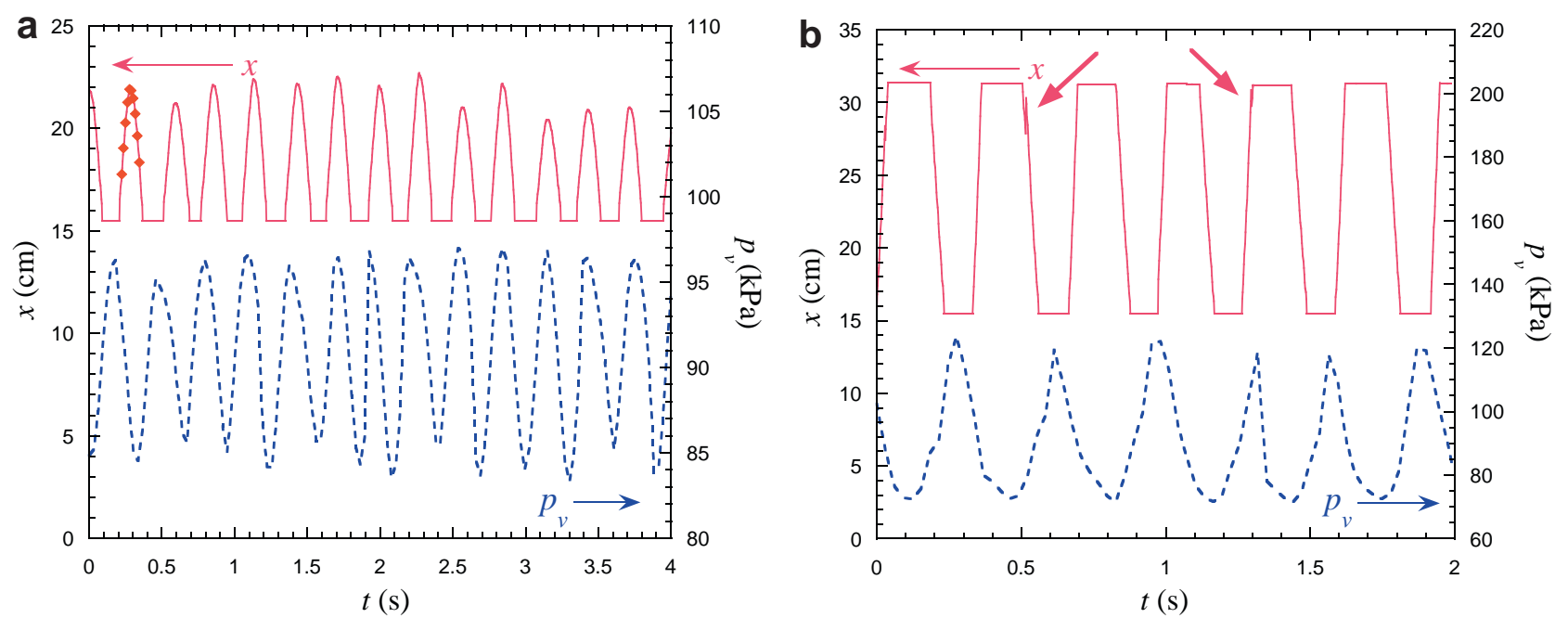

Fig. 2. Measured $x$ and $p_{\mathrm{v}}$ evolutions for $p_{\mathrm{r}}=90 \mathrm{kPa}$ and (a) $T_{\mathrm{e}}=45^{\circ} \mathrm{C}, T_{\mathrm{C}}=10^{\circ} \mathrm{C}$; the solid characters show the time moments at which the snapshots in Fig. 3 were taken. (b) $T_{\mathrm{e}}=65^{\circ} \mathrm{C}, T_{\mathrm{C}}=0^{\circ} \mathrm{C}$. The slanted arrows indicate the kinks described in the text.

shows oscillations of the meniscus and the pressure $p_{\mathrm{v}}$ of the vapor close to the instability threshold. Reservoir pressure $p_{\mathrm{r}}$ is $90 \mathrm{kPa}$. The amplitude of pressure is about $15 \mathrm{kPa}$. The displacement curve is truncated from below because the adiabatic and evaporator sections are opaque and the meniscus displacement cannot be measured inside them. The evolution is not exactly periodic; the average period is about $0.28 \mathrm{~s}$. Note the intermittency in the height of the minima of the pressure curve: each second or third minimum is higher than the others.

For $T_{\mathrm{e}}=65^{\circ} \mathrm{C}$ and $T_{\mathrm{c}}=0{ }^{\circ} \mathrm{C}$, the maximum meniscus displacement is about $39 \mathrm{~cm}$. The displacement variation shown in Fig. $2 \mathrm{~b}$ is truncated not only from below but also from above because of the limited field of view of the camera. Unlike the previous case, the pressure variation is regular (see Fig. 2b). The amplitude of pressure is about $50 \mathrm{kPa}$. The pressure curve is steeper at expansion than at compression. The oscillation period is about $0.34 \mathrm{~s}$. Kinks in the displacement plot correspond to the sudden relocation of the meniscus because of the following effect. When the rate of condensation onto the moving liquid film in the condenser is high enough, the liquid film becomes unstable. It means that the liquid film bulges toward the tube axis and coalesces with the bulged liquid film from the opposite wall. This leads to the formation of a secondary small bubble and the meniscus of the main bubble is relocated. This can occur during the movement both towards or away from the evaporator. The secondary bubble disappears quickly by coalescing with the main bubble, which causes another kink.

Fig. 3 shows a series of images corresponding to Fig. 2a. Dark part of the capillary tube to the left is the vapor bubble and the bright part to the right is the liquid plug.

Note that in both cases the meniscus penetrates into the evaporator at each oscillation. In Section 4 we discuss a comparison of the experiment with the model presented below.

\section{Theoretical and numerical modelling}

\subsection{Model geometry}

The geometry of the problem (Fig. 4) is similar to the works presented earlier in $[11,12]$. The liquid plug confines a vapor bubble 

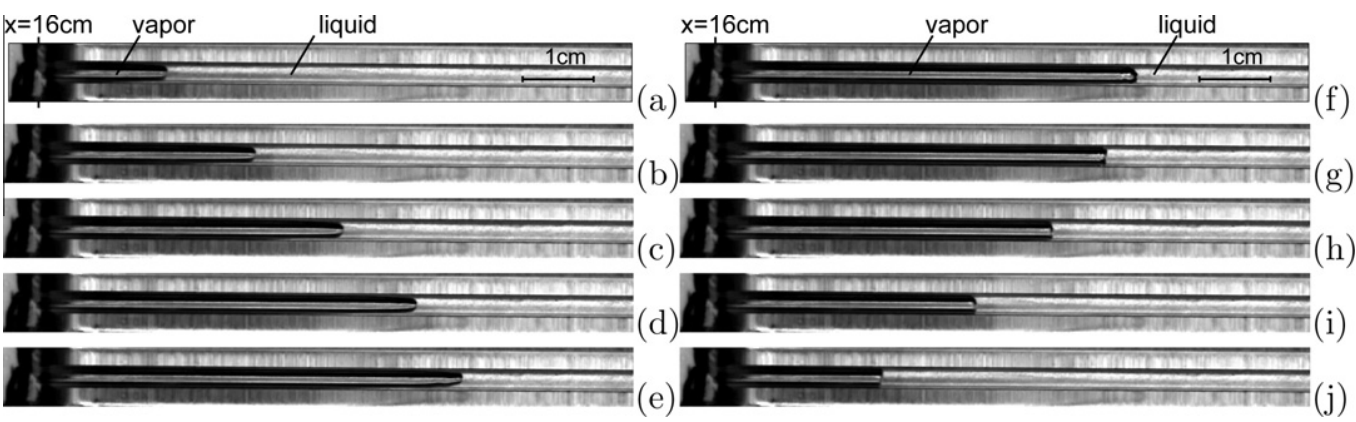

Fig. 3. Consecutive snapshots of the transparent condenser showing the meniscus position for one cycle $\left(T_{\mathrm{e}}=45^{\circ} \mathrm{C}, T_{\mathrm{C}}=10^{\circ} \mathrm{C}\right.$ and $\left.p_{\mathrm{r}}=90 \mathrm{kPa}\right)$. Time interval between two consecutive images is $13.3 \mathrm{~ms}$. The liquid reservoir is to the right, and the evaporator is to the left of the images.

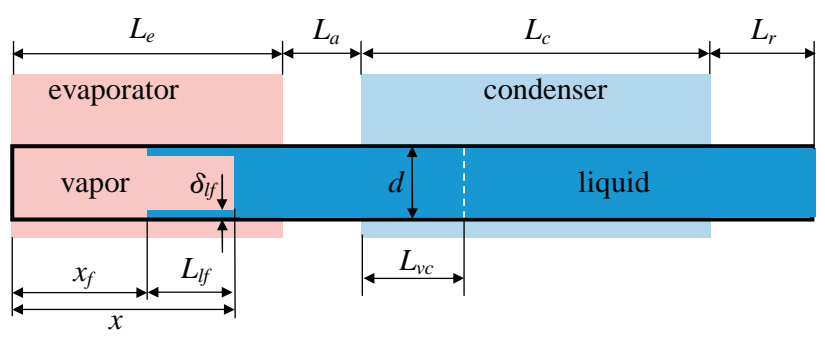

Fig. 4. Schematic of the model. The white dashed line represents the position of the meniscus when it is situated in the condenser. A constant pressure $p_{\mathrm{o}}$ is imposed at the open right end of the tube.

(of the same substance) at the closed end of a cylindrical tube of diameter $d$ and cross-section area $S=\pi d^{2} / 4$. The pressure $p_{\mathrm{o}}$ at the right open end of the tube is constant and assumed to be equal to the sum of the reservoir pressure $p_{\mathrm{r}}$ and the hydrostatic pressure of the liquid column in the reservoir. This geometry is also equivalent to a half of the U-shaped tube, provided that the oscillations in its branches are symmetrical. The evaporator length is $L_{\mathrm{e}}$ and the evaporator tube wall is maintained at a constant temperature $T_{\mathrm{e}}$. This section is separated from the condenser of length $L_{\mathrm{c}}$ by an adiabatic section of length $L_{\mathrm{a}}$. The condenser wall temperature is constant and equal to $T_{\mathrm{c}}$. The vapor bubble is characterized by its length $x$, the vapor temperature $T_{\mathrm{v}}$ and the pressure $p_{\mathrm{v}}$. The evaporation/condensation occurs mainly from/to the liquid film, the part of the length of which inside the evaporator is denoted by $L_{\mathrm{lf}}$. The length of the dry part of evaporator is denoted by $x_{\mathrm{f}}$. A part $L_{\mathrm{r}}$ of the liquid plug that extends beyond the condenser is much larger than $L_{\mathrm{t}}=L_{\mathrm{e}}+L_{\mathrm{a}}+L_{\mathrm{c}}$.

\subsection{Superheated vapor model}

Before considering the full model that accounts for the film evaporation, we first analyze a simpler model with no film $\left(L_{\mathrm{lf}}=0\right)$. This is done in an attempt to obtain some analytical results. Within several details, it is analogous to the single-bubble version of the model presented in [13]. By the reasons mentioned above, this model is also similar to that of Dobson [11,12] (at least it coincides with it in the linear approximation treated below).

The first principle of thermodynamics written for the vapor bubble (vapor energy equation) is

$c_{\mathrm{vv}} \frac{\mathrm{d}\left(m_{\mathrm{v}} T_{\mathrm{v}}\right)}{\mathrm{d} t}=U_{\mathrm{e}} \pi d L_{\mathrm{ve}}\left(T_{\mathrm{e}}-T_{\mathrm{v}}\right)-U_{\mathrm{c}} \pi d L_{\mathrm{vc}}\left(T_{\mathrm{v}}-T_{\mathrm{c}}\right)-p_{\mathrm{v}} S V$,

where $c_{\mathrm{vv}}$ is the vapor specific heat at constant volume assumed to be independent of the temperature; $m_{\mathrm{v}}$ : vapor mass; $U_{\mathrm{e}}$ and $U_{\mathrm{c}}$ : heat exchange coefficients of evaporation and condensation, respectively. The heat exchange terms come from evaporation/condensation heat exchange, see [11-13]. These terms are equivalent to those of the sensible heat exchange of the gas with the tube walls. The last term corresponds to the mechanical work.

The meniscus velocity is defined by the equation:

$\dot{x}=V$

where dot means the time derivative. The lengths of the vapor in contact with the evaporator and the condenser are defined by the expressions:

$L_{\mathrm{ve}}= \begin{cases}x & x \leqslant L_{\mathrm{e}} \\ L_{\mathrm{e}} & \text { otherwise. }\end{cases}$

$L_{\mathrm{vc}}= \begin{cases}0 & x<L_{\mathrm{e}}+L_{\mathrm{a}}, \\ x-\left(L_{\mathrm{e}}+L_{\mathrm{a}}\right) & L_{\mathrm{e}}+L_{\mathrm{a}} \leqslant x<L_{\mathrm{e}}+L_{\mathrm{a}}+L_{\mathrm{c}}, \\ L_{\mathrm{c}} & \text { otherwise. }\end{cases}$

The vapor pressure is defined by the equation of state which is assumed to be that of ideal gas like in [11-13]:

$p_{\mathrm{v}}=\frac{m_{\mathrm{v}} R_{\mathrm{v}} T_{\mathrm{v}}}{S x}$

where $R_{\mathrm{V}}$ is the gas constant divided by the vapor molar mass. This equation can be applied because the vapor is assumed to be superheated, which means that its temperature is larger than the saturation temperature $T_{\mathrm{sat}}\left(p_{\mathrm{v}}\right)$. Note that $m_{\mathrm{v}}$ is constant in the superheated vapor model and is defined by the initial conditions. The momentum equation for the liquid plug reads:

$\frac{\mathrm{d}\left(V m_{\mathrm{l}}\right)}{\mathrm{d} t}=F_{\mathrm{p}}-F_{\mathrm{f}} \operatorname{sign}(V)$,

where

$m_{1}=\rho\left(L_{\mathrm{t}}+L_{\mathrm{r}}-x\right) S$

is the liquid mass; $\rho$ is the liquid density. $F_{\mathrm{p}}=\left(p_{\mathrm{v}}-p_{\mathrm{o}}\right) S$ is the pressure force and

$F_{\mathrm{f}}=\frac{1}{2} C_{\mathrm{f}} d \rho \pi\left(L_{\mathrm{t}}+L_{\mathrm{r}}-x\right) V^{2}$,

is the friction force. Like in [11], the coefficient $C_{\mathrm{f}}$ is taken for the single-phase flow. It depends on the Reynolds number $\operatorname{Re}=V d / v$ $(v$ : kinematic viscosity):

$C_{f}= \begin{cases}0 & R e=0, \\ 16 & R e<1, \\ 16 / R e & 1 \leqslant \operatorname{Re}<1180, \\ 0.078 \operatorname{Re}^{-0.25} & R e \geqslant 1180 .\end{cases}$

The linear stability analysis of this model can be performed as usually [17]. The following substitutions are thus made: 
$T_{\mathrm{v}}=\left\langle T_{\mathrm{v}}\right\rangle\left(1+y_{1}\right)$

$x=\langle x\rangle\left(1+y_{2}\right)$,

$V=y_{3}\langle x\rangle / \tau$,

where $\tau$ is a characteristic time scale and $y_{\mathrm{i}}(t) \ll 1, i=1,2,3$ are infinitesimally small perturbations of the constant equilibrium values (denoted by the angle brackets). It should be checked if the initial perturbations grow or decay in time and under which conditions. In other words, one needs to determine if the equilibrium is stable or not.

It turns out that the form of set of equations linearized with respect to $y_{\mathrm{i}}$ changes depending on the equilibrium meniscus position $\langle x\rangle$. Two cases need to be considered separately, where $\langle x\rangle$ is located in (i) the evaporator or adiabatic section or (ii) the condenser. Note that due to the smallness of oscillations around the equilibrium, the meniscus can be considered to always stay in the same section as $\langle x\rangle$.

\subsubsection{Equilibrium meniscus position located in the evaporator or} adiabatic sections

For this case $\langle x\rangle\left\langle L_{\mathrm{e}}+L_{\mathrm{a}}, L_{\mathrm{vc}}=0\right.$, and all the condenser parameters disappear from the problem. By zeroing the time derivatives, one obtains from (1), (2), (5), (6):

$$
\begin{aligned}
& \left\langle T_{\mathrm{v}}\right\rangle=T_{\mathrm{e}}, \\
& \left\langle p_{\mathrm{v}}\right\rangle=p_{\mathrm{o}}, \\
& \langle x\rangle=\frac{m_{\mathrm{v}} R_{\mathrm{v}}\left\langle T_{\mathrm{v}}\right\rangle}{S p_{\mathrm{o}}} .
\end{aligned}
$$

Since the competition between evaporation and condensation is nonexistent in this case, it is a priori clear that the equilibrium is stable for any parameters. It can also be shown rigorously. By using (9)-(14) in (1), (2), (5), (6) and by linearizing them with respect to $y_{\mathrm{i}}$, one obtains the set:

$$
\begin{aligned}
& \dot{y_{1}}=-\alpha y_{1}-\beta y_{2}-K y_{3}, \\
& \dot{y_{2}}=y_{3}, \\
& \dot{y}_{3}=y_{1}-y_{2},
\end{aligned}
$$

where

$$
\begin{aligned}
K & =\frac{R_{\mathrm{v}}}{c_{\mathrm{vv}}}, \\
\alpha & =\frac{\tau}{t_{\mathrm{c} 1}}>0 \\
\beta & =0 \\
\tau & =\sqrt{\frac{\rho\left(L_{\mathrm{t}}+L_{\mathrm{r}}-\langle x\rangle\right)\langle x\rangle}{p_{o}}} \\
t_{\mathrm{c} 1} & =\frac{m_{\mathrm{v}} c_{\mathrm{vv}}}{\pi d U_{\mathrm{c}}\langle x\rangle} .
\end{aligned}
$$

The expression (22) is valid for the case $\langle x\rangle<L_{\mathrm{e}}$. For the case $L_{\mathrm{e}}<\langle x\rangle\left\langle L_{\mathrm{e}}+L_{\mathrm{a}},\langle x\rangle\right.$ in (22) should be replaced by $L_{\mathrm{e}}$. One mentions that the characteristic time $2 \pi / \tau$ corresponds to the eigenfrequency of a massive plug that oscillates in a cylinder closed from one side and filled with a gas.

The linear stability analysis shows that the system is always stable in this case. The relaxation time is given by (22). The relaxation is the fastest when the following simple relation is satisfied: $\alpha=1+K / 2$. An example of the numerical solution of the full (nonlinearized) set of Eqs. 1, 2, 5, 6 for this case is shown in Fig. 5a. The calculations are performed using the parameters for water at 1 bar [11] and $T_{\mathrm{e}}=150^{\circ} \mathrm{C}, T_{\mathrm{c}}=25^{\circ} \mathrm{C}, L_{\mathrm{e}}=6 \mathrm{~cm}, L_{\mathrm{a}}=4 \mathrm{~cm}, L_{\mathrm{c}}=5 \mathrm{~cm}$, $L_{\mathrm{r}}=4.5 \mathrm{~cm}, U_{\mathrm{e}}=U_{\mathrm{c}}=800 \mathrm{~W} /\left(\mathrm{m}^{2} \mathrm{~K}\right)$. In agreement with the analytical analysis, the system remains stable and the oscillations, which appear due to initial deviation from equilibrium, quickly decline. The meniscus position tends to the asymptotic value defined by (14). Note that a temporary penetration of the meniscus into the condenser does not make the system unstable.

3.2.2. Equilibrium meniscus position located in the condenser section In this case $L_{\mathrm{ve}}=L_{\mathrm{e}}, L_{\mathrm{vc}}=x-L_{\mathrm{e}}-L_{\mathrm{a}}$ and

$$
\begin{gathered}
\left\langle T_{\mathrm{v}}\right\rangle=\left\{\left(\xi U_{\mathrm{c}} T_{\mathrm{c}}+U_{\mathrm{c}}\left(L_{\mathrm{a}}+L_{\mathrm{e}}\right)-L_{\mathrm{e}} U_{\mathrm{e}}\right)+\left[\left(\xi U_{\mathrm{c}} T_{\mathrm{c}}+U_{\mathrm{c}}\left(L_{\mathrm{a}}+L_{\mathrm{e}}\right)\right.\right.\right. \\
\left.\left.\left.-L_{\mathrm{e}} U_{\mathrm{e}}\right)^{2}+4 \xi U_{\mathrm{c}}\left(U_{\mathrm{e}} L_{\mathrm{e}} T_{\mathrm{e}}-U_{\mathrm{c}}\left(L_{\mathrm{a}}+L_{\mathrm{e}}\right) T_{\mathrm{c}}\right)\right]^{1 / 2}\right\}\left(2 \xi U_{\mathrm{c}}\right)^{-1}
\end{gathered}
$$

where $\xi=m_{\mathrm{v}} R_{\mathrm{v}} / S p_{\mathrm{o}}$. Eqs. (13), (14), where $\left\langle T_{\mathrm{v}}\right\rangle$ is now defined by (23), remain valid.

The set of Eqs. (15)-(17) remains the same as in the previous case, but the values of the following parameters change: $\alpha=\tau$ / $t_{\mathrm{c} 2}>0$ and $\beta=\tau / t_{\mathrm{c} 3}>0$, where
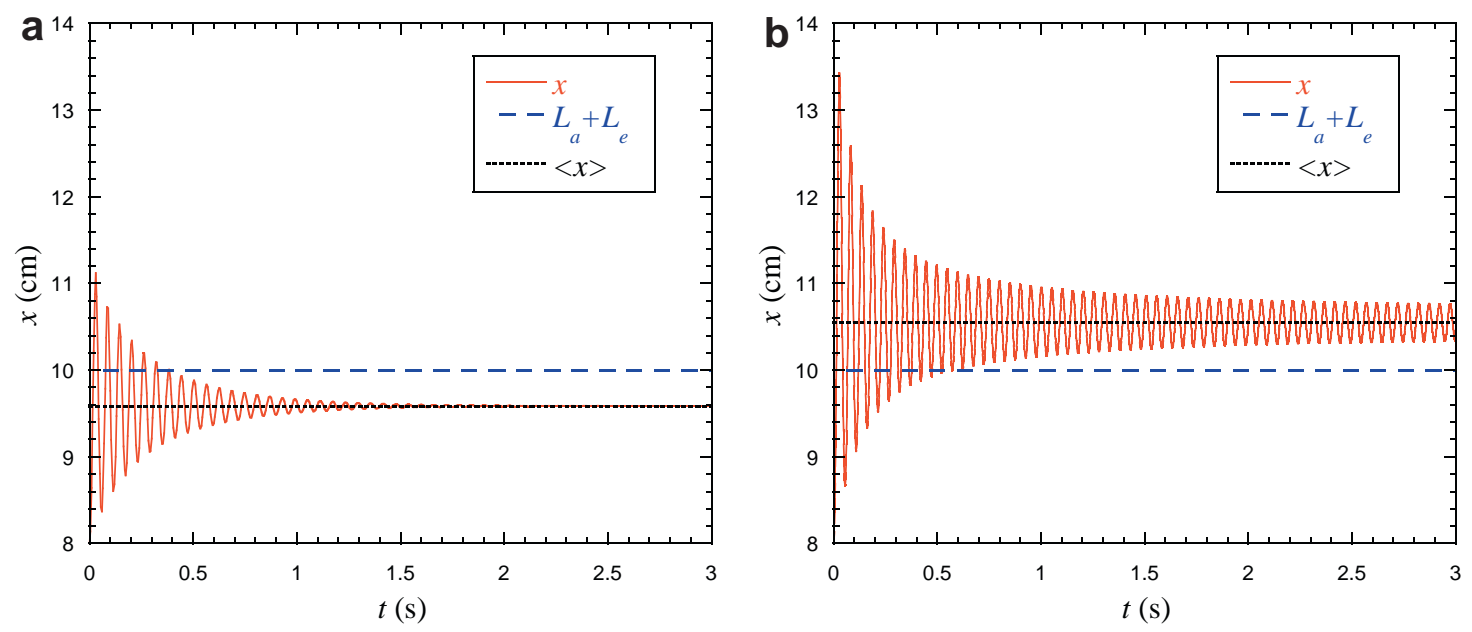

Fig. 5. Meniscus displacement calculated within the superheated vapor model. (a) $\langle x\rangle\left\langle L_{\mathrm{e}}+L_{\mathrm{a}}\right.$ case calculated with the following initial conditions at: $t=0$, $T_{\mathrm{v}}=80{ }^{\circ} \mathrm{C}$, $x=8 \mathrm{~cm}, V=0, p_{\mathrm{v}}=p_{\mathrm{o}}$. (b) $\langle x\rangle>L_{\mathrm{e}}+L_{\mathrm{a}}$ case calculated for $T_{\mathrm{v}}(t=0)=40^{\circ} \mathrm{C}$. Other parameters remain unchanged from the case (a). 
$t_{\mathrm{c} 2}=\frac{m_{\mathrm{v}} c_{\mathrm{vv}}}{\pi d\left[L_{\mathrm{e}} U_{\mathrm{e}}+U_{\mathrm{c}}\left(\langle x\rangle-L_{\mathrm{a}}-L_{\mathrm{e}}\right)\right]}, \quad t_{\mathrm{c} 3}=\frac{m_{\mathrm{v}} c_{\mathrm{vv}}}{\pi d U_{\mathrm{c}}\langle x\rangle\left(1-T_{\mathrm{c}} /\left\langle T_{\mathrm{v}}\right\rangle\right)}$

It can be shown that in this case the system may exhibit either stable or unstable behavior. More specifically, the system develops an instability if $\beta / \alpha>K$ or, equivalently:

$\frac{U_{\mathrm{c}}\langle x\rangle\left(1-T_{\mathrm{c}} /\left\langle T_{\mathrm{v}}\right\rangle\right)}{L_{\mathrm{e}} U_{\mathrm{e}}+U_{\mathrm{c}}\left(\langle x\rangle-L_{\mathrm{a}}-L_{\mathrm{e}}\right)}>\frac{R_{\mathrm{v}}}{C_{\mathrm{vv}}}$.

A corresponding solution of the nonlinear set (1), (2), (5), (6) is shown in Fig. 5b. For this case, $\beta=3.8, \alpha=8.4, \beta / \alpha>K=0.32$ and the instability should occur. Indeed, the meniscus oscillates around a position given by (14), (23).

Note that the amplitude of oscillations is small. After a transition period, the meniscus always remains in the condenser and adiabatic sections without penetrating into the evaporator. In the framework of the above cited models, where the heat exchange terms come from the evaporation/condensation arguments, it means that the evaporation is absent, which is incoherent. According to our parametric analysis, this seems to be a general feature of the superheated vapor type models. Dobson [11] reports an intermittent penetration of the meniscus into the evaporator. In our attempt of reproducing his results with his model and his parameters we could not obtain such a penetration and the meniscus always remained in the condenser. We attribute this discrepancy to a poor stability of the numerical (explicit Euler) method used by Dobson to solve his equations. Throughout this paper we use the fourth order Runge-Kutta method which is well known to be stable. The computations are performed in double precision with a $\mathrm{C}++$ numerical code.

The analytical expression for the oscillation period $P$ can be found analytically at the instability threshold corresponding to $\beta /$ $\alpha=K$ :

$P=2 \pi \frac{\tau}{\sqrt{1+K}}$

A numerical analysis shows that the oscillation period is always very close to (25).

The instability mechanism in such a model is simple. When the meniscus moves into the condenser section ( $x$ increases), the pressure decreases not only because the gas expands but also because it is cooled down by the increasingly strong heat exchange with the cold part of the tube (a part of the vapor bubble is in the condenser). Similarly, during the backward motion of the meniscus, the gas is heated up not only because of the compression, but also because of the transfer of the heat from the hot portion of the walls. This pumping of energy into the gas acts in phase with the oscillations and thus increases them. Their amplitude cannot however be strong; except for the friction force, it is limited by the interplay of heat exchange rates with evaporator and condenser. Indeed, the calculation shows that the amplitude remains finite even if the friction force $F_{\mathrm{f}}$ is zeroed.

We conclude the analysis of the superheated vapor model. In its main lines, it corresponds to the PHP models of other authors, at least in the linear approximation. The criterion of linear instability is established in terms of the system parameters. It is shown that such a model can develop only small amplitude oscillations during which the liquid-vapor meniscus remains inside the condenser without penetrating into the evaporator. This means that the evaporation can hardly be described by such a model. In the next section, a new model is proposed.

\subsection{Film evaporation/condensation model}

The present approach inherits from two existing classes of models. On one hand, it is the approach [16] that stresses the impor- tance of the thin liquid films shown schematically in Fig. 4 However, it considers the vapor to be isothermal at saturation temperature $T_{\text {sat }}$ for the current vapor pressure $p_{\mathrm{v}}$. In our opinion, it is hardly possible because the vapor compression or expansion due to the liquid plug motion can lead to the vapor bulk temperature different from the interface (saturation) temperature. On the other hand, there is an approach [11-13] that does describe the vapor temperature rise due to the vapor compression but ignores the interface saturation temperature.

In the present model, the main (bulk) part of the vapor is isothermal. Its temperature $T_{\mathrm{v}}$ is allowed to be both higher (due to compression) and lower (due to expansion) than the saturation temperature that occurs at the vapor-liquid interface. In the latter case the vapor is in the metastable state which may persist only during short periods of time. Thin boundary layers form in the vapor near its borders. A difference between the interface and bulk vapor temperatures occurs because of the weakness of the heat diffusion in the vapor. The order of value of the thickness $\delta_{\mathrm{bl}}$ of the temperature boundary layer in the vapor can be estimated by the expression $\delta_{\mathrm{bl}}=\sqrt{P D} \approx 1 \mathrm{~mm}$ where $D \approx 4 \mathrm{~mm}^{2} / \mathrm{s}$ is the thermal diffusivity of the vapor and $P \approx 0.3 \mathrm{~s}$ is the oscillation period. Since the vapor bubble is much longer, the temperature inside it can differ from that of the meniscus.

Like in the above cited models, the heat exchange inside the liquid turns out to be decoupled from the meniscus dynamics part of the problem and is not considered here.

The film is assumed to be of the constant thickness $\delta_{\text {lf }}[11,12]$. It completely covers the tube walls in the condenser and adiabatic sections (when the vapor exists in them) but may be partially or completely evaporated in the evaporator. Thus only a part of the film situated in the evaporator (Fig. 4) matters. The dynamics of its length $L_{\text {lf }} \geqslant 0$ is described by the following equation:

$$
\frac{\mathrm{d} L_{\mathrm{lf}}}{\mathrm{d} t}= \begin{cases}0 & \text { if } L_{\mathrm{lf}}=0, \quad V<0 \quad \text { and } x \leqslant L_{\mathrm{e}} \\ -\dot{m}_{\mathrm{e}}^{\mathrm{f}} /\left(\rho \pi d \delta_{\mathrm{lf}}\right) & \text { if } x>L_{\mathrm{e}}, \\ V-\dot{m}_{\mathrm{e}}^{\mathrm{f}} /\left(\rho \pi d \delta_{\mathrm{lf}}\right) & \text { otherwise. }\end{cases}
$$

This expression means that the film is left by the liquid plug when it recedes $(V>0)$ and is "eaten up" when it advances $(V<0)$. This term is needed only when the meniscus is inside the evaporator (i.e. when $x \leqslant L_{\mathrm{e}}$ ). This description has been introduced by Dobson [11,12] and is justified by the slow hydrodynamic response of viscous films. The first line of (26) is added to avoid negative $L_{\text {lf }}$ values when the film disappears during the liquid plug advance. The second line and a part of the third line are similar and mean film length variation due to film evaporation or possibly condensation in the evaporator with the mass rate $\dot{m}_{\mathrm{e}}^{\mathrm{f}}$ (positive at evaporation, negative at condensation).

The heat balance at the film vapor-liquid interface defines the mass exchange rate. One can write the heat balances separately for the evaporator and the condenser:

$h_{\mathrm{lv}} \dot{m}_{\mathrm{e}}^{\mathrm{f}}=U_{\mathrm{e}} \pi d L_{\mathrm{lf}}\left[T_{\mathrm{e}}-T_{\mathrm{sat}}\left(p_{\mathrm{v}}\right)\right]$,

$h_{\mathrm{lv}} \dot{m}_{\mathrm{c}}^{\mathrm{f}}=U_{\mathrm{c}} \pi d L_{\mathrm{vc}}\left[T_{\mathrm{c}}-T_{\mathrm{sat}}\left(p_{\mathrm{v}}\right)\right]$,

where $h_{\mathrm{lv}}$ is the latent heat of vaporization. The r.h.s. of each equation corresponds to the heat flux in the film. It is proportional to the difference of the wall and interface temperatures. The heat flux in the vapor is assumed to be negligible because of its low heat conductivity. The heat transfer coefficients are defined by the film thickness. Since it is supposed to be the same in the evaporator and in the condenser, $U_{\mathrm{e}}=U_{\mathrm{c}}=\gamma k_{\mathrm{l}} / \delta_{\mathrm{lf}}$ where $\gamma \leqslant 1$ is a coefficient accounting for the spatial variation of the film thickness. $L_{\mathrm{vc}}$ is defined in the previous section. 
Condensation might occur in the evaporator because of the vapor compression. We assume implicitly in (27) that the condensation on the dry wall is much smaller in comparison with that on the film (because of the nucleation threshold which, for partial wetting case, is large on the wall but zero on the liquid). The condensation area in (27) is thus $\pi d L_{1 f}$, the same as that of evaporation.

Although much weaker than at the film interface, evaporation and condensation occur at the remaining meniscus part (other than the film) and exist even if the film is evaporated completely. The heat balance on the meniscus depends on whether the meniscus situates inside the evaporator or the condenser:

$h_{\mathrm{lv}} \dot{m}_{\mathrm{e}}^{\mathrm{m}}=U_{\mathrm{e}}^{\mathrm{m}} \pi d L_{\mathrm{e}}^{\mathrm{m}}\left[T_{\mathrm{e}}-T_{\mathrm{sat}}\left(p_{\mathrm{v}}\right)\right]$,

$h_{\mathrm{lv}} \dot{m}_{\mathrm{c}}^{\mathrm{m}}=U_{\mathrm{c}}^{\mathrm{m}} \pi d L_{\mathrm{c}}^{\mathrm{m}}\left[T_{\mathrm{c}}-T_{\mathrm{sat}}\left(p_{\mathrm{v}}\right)\right]$.

The lengths that enter the above equations are defined so as to account for the exact location of the meniscus:

$L_{\mathrm{e}}^{\mathrm{m}}= \begin{cases}L_{\mathrm{m}}, & x \leqslant L_{\mathrm{e}}, \\ 0, & \text { otherwise },\end{cases}$

$L_{\mathrm{c}}^{\mathrm{m}}= \begin{cases}L_{\mathrm{m}}, & L_{\mathrm{e}}+L_{\mathrm{a}} \leqslant x<L_{\mathrm{e}}+L_{\mathrm{a}}+L_{\mathrm{c}}, \\ 0, & \text { otherwise. }\end{cases}$

The length $L_{\mathrm{m}}$ is assumed to be of the order of $1 \%$ of the tube radius and the heat exchange coefficients $U_{\mathrm{e}}^{\mathrm{m}}=U_{\mathrm{c}}^{\mathrm{m}}$ are assumed to be about $0.3 \mathrm{U}$. We note that at normal operating conditions, the contribution from the meniscus to the overall evaporation should be very small with respect to that of the film and can be non-negligible only for very high $T_{\mathrm{e}}$.

The vapor mass change is

$\dot{m}_{\mathrm{v}}=\dot{m}_{\mathrm{e}}^{\mathrm{f}}+\dot{m}_{\mathrm{c}}^{\mathrm{f}} h+\dot{m}_{\mathrm{e}}^{\mathrm{m}}+\dot{m}_{\mathrm{c}}^{\mathrm{m}}$.

Instead of (1) for the superheated vapor model, the vapor heat balance reads:

$m_{\mathrm{v}} c_{\mathrm{vv}} \dot{T}_{\mathrm{v}}=\dot{m}_{\mathrm{v}} R_{\mathrm{v}} T+q_{\text {sens }}-p_{\mathrm{v}} S V$,

where the sensible heat exchange of the tube with the vapor in the evaporator is accounted for by the term:

$q_{\text {sens }}=U_{\mathrm{v}} \pi d x_{\mathrm{f}}\left(T_{\mathrm{e}}-T_{\mathrm{v}}\right)$.

Note a mistake in the works $[11,12]$ in the heat balance equation. Its correct form (32) is taken from [13].

Eq. (33) involves the length of the dry part of the evaporator (Fig. 4):

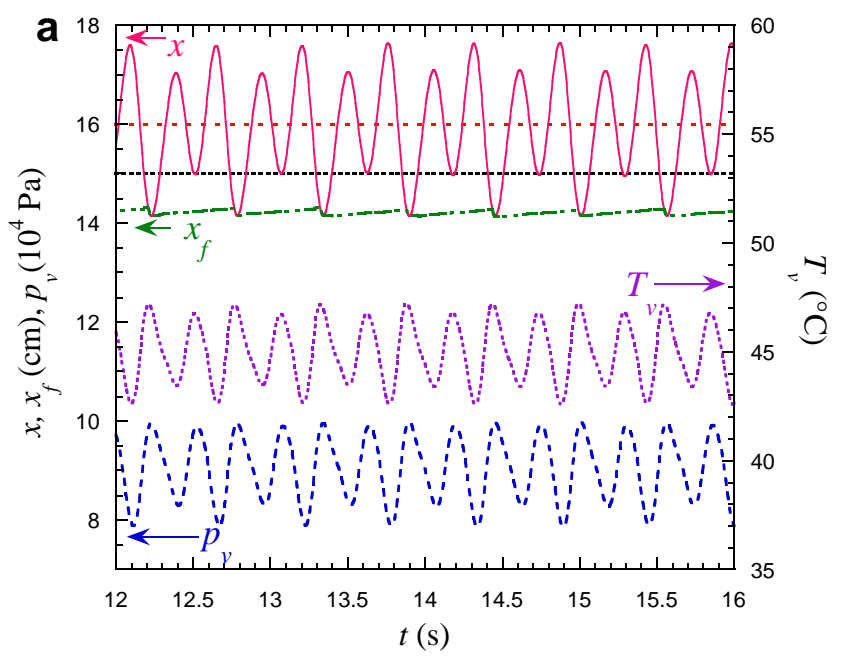

$x_{\mathrm{f}}= \begin{cases}x-L_{\mathrm{lf}}, & x<L_{\mathrm{e}}, \\ L_{\mathrm{e}}-L_{\mathrm{lf}}, & \text { otherwise }\end{cases}$

and the heat exchange coefficient $U_{\mathrm{v}}$. The sensible heat exchange with the vapor is much weaker than the evaporation/condensation exchange and is included for completeness only. The estimated above thickness $\delta_{\mathrm{bl}}$ of the diffusion boundary layer is used for the estimation $U_{\mathrm{v}}=k_{\mathrm{v}} / \delta_{\mathrm{b}}$, where $k_{\mathrm{v}}$ is the heat conductivity of the vapor.

Based upon the experimental observations, it is assumed that when the vapor penetrates inside the adiabatic section and the condenser, the liquid film always covers the internal walls. There is no direct contact and thus no sensible heat exchange between the vapor and the condenser.

The expressions (2), (4)-(8), (26)-(34) fully define a set of five ordinary differential Eqs. (2), (6), (26), (31), (32) that govern the system.

It is easy to check that the linearization of this system does not make any sense; an analytical study is thus impossible and a numerical solution needs to be found. The set is solved with the standard fourth order Runge-Kutta method.

For the purpose of the comparison with the experimental setup described above, two different values of $L_{\mathrm{r}}, L_{\mathrm{r}}^{\text {eff, } \mathrm{i}}$ and $L_{\mathrm{r}}^{\text {eff, } \mathrm{f}}$ were used in the expressions (7) and (8) respectively. $L_{\mathrm{r}}^{\text {eff,i }}$ accounts for the fluid inertia and thus enters the expression for the period of oscillations (25) through the time $\tau(21)$. Note that (25) describes correctly the period of the simulated oscillations. $L_{\mathrm{r}}^{\text {eff,i }}$ has been determined by fitting (25) to the experimental data on the period of oscillations. $L_{\mathrm{r}}^{\text {eff } f}$ defines the hydrodynamic friction in the system. Note that the friction force (8) used in the existing PHP modelling is quite a rough model of the reality. Such an expression corresponds to the viscous friction in the constant velocity flow of a single-phase fluid. The existence of two-phase flow (in particular, the thin film flow) can lead to a drastic increase of the friction so that the real friction force is much larger than that predicted by (8). Indeed it is well known [18] that the viscous friction that occurs in the vicinity of the junction of the moving meniscus with the film or dry wall can be extremely large. This means that the experimental fit of $L_{\mathrm{r}}^{\text {eff, }}$ might result in a much larger value than $L_{\mathrm{t}}$. Two examples of the numerical simulation results are shown in Fig. 6. They use $n$-pentane as the working fluid whose material parameters are shown in Table 1a. $p_{\mathrm{o}}=90.6 \mathrm{kPa}$ accounts for the hydrostatic pressure of $10 \mathrm{~cm}$ high liquid column. The following expression [19] has been used for the saturation curve

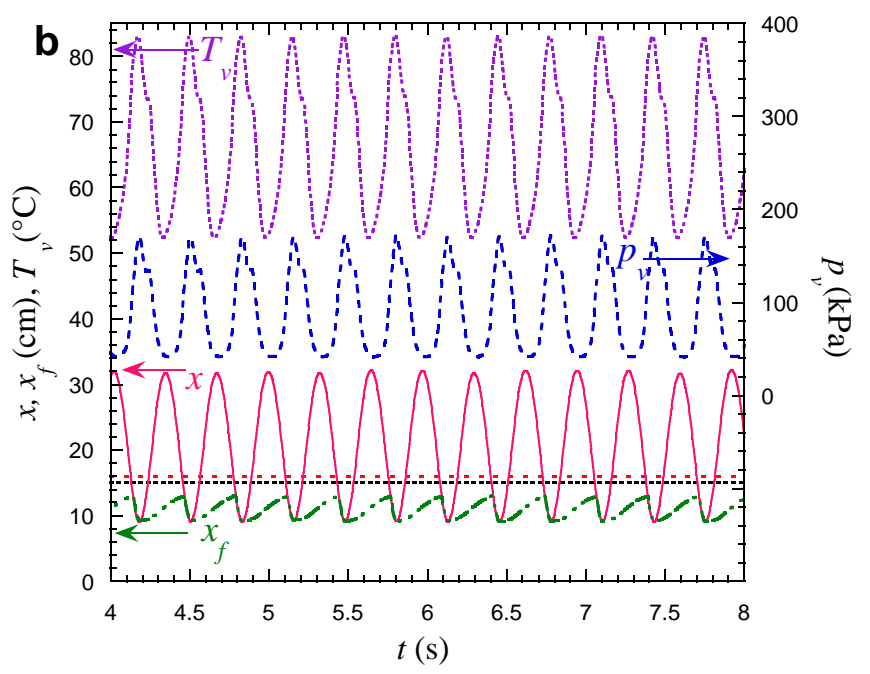

Fig. 6. Simulation results, to be compared with Fig. 2. The evolution of $p_{\mathrm{v}}, T_{\mathrm{v}}, x$ and $x_{\mathrm{f}}$ are shown. The lower and upper horizontal lines correspond to the ends of the evaporator $\left(L_{\mathrm{e}}=15 \mathrm{~cm}\right)$ and adiabatic sections $\left(L_{\mathrm{e}}+L_{\mathrm{a}}=16 \mathrm{~cm}\right)$, respectively. (a) $T_{\mathrm{e}}=45^{\circ} \mathrm{C}, T_{\mathrm{C}}=10^{\circ} \mathrm{C}$; (b) $T_{\mathrm{e}}=65^{\circ} \mathrm{C}, T_{\mathrm{C}}=0{ }^{\circ} \mathrm{C}$. 
Table 1a

Values of $n$-pentane parameters used to obtain Fig. $6 a$ and $b$.

\begin{tabular}{lcl}
\hline Quantity & Value & Units \\
\hline$h_{\mathrm{lv}}$ & 357.7 & $\mathrm{~kJ} / \mathrm{kg}$ \\
$k_{\mathrm{l}}$ & 120 & $\mathrm{~mW} /(\mathrm{m} \mathrm{K})$ \\
$k_{\mathrm{v}}$ & 22 & $\mathrm{~mW} /(\mathrm{m} \mathrm{K})$ \\
$c_{\mathrm{vv}}$ & 1685 & $\mathrm{~J} /(\mathrm{kg} \mathrm{K})$ \\
$R_{\mathrm{v}}$ & 115 & $\mathrm{~J} /(\mathrm{kg} \mathrm{K})$ \\
$\rho$ & 615.8 & $\mathrm{~kg} / \mathrm{m}^{3}$ \\
$v$ & 0.385 & $\mathrm{~mm}^{2} / \mathrm{s}$ \\
\hline
\end{tabular}

$$
\begin{aligned}
T_{\text {sat }}\left(p_{\mathrm{v}}\right) & =\exp \left[5.059+0.039167 \log p_{\mathrm{v}}+0.0012452\left(\log p_{\mathrm{v}}\right)^{2}\right. \\
& \left.-5.3621 \cdot 10^{-5}\left(\log p_{\mathrm{v}}\right)^{3}+7.9644 \cdot 10^{-6}\left(\log p_{\mathrm{v}}\right)^{4}\right]
\end{aligned}
$$

where $p_{\mathrm{v}}$ in Pa and $T_{\mathrm{v}}$ is in K. Several parameters (Table $1 \mathrm{~b}$ ) that could not be measured in the experiment were adjusted to fit the experimental data of Fig. 2.

It has been checked that the ideal gas equation of state is applicable as $T_{\mathrm{v}}$ remains to be larger and far enough from $T_{\text {sat }}$ throughout the simulations. The vapor superheat is about $12 \mathrm{~K}$ for Fig. $6 \mathrm{a}$ and $30 \mathrm{~K}$ for Fig. $6 \mathrm{~b}$.

In addition, a parametric analysis has been carried out. It has shown that, unlike the superheated vapor model, the system is robust: it is almost insensitive to the initial conditions. Independently of them (in reasonable limits), the established oscillations are of the same amplitude, average value and frequency. It means that the system itself chooses the mass of the vapor.

While the system is almost insensible to the value of $L_{\mathrm{m}}$, the other parameters of Table $1 \mathrm{~b}$ can strongly influence its behavior. As expected, $L_{r}^{\text {eff,i }}$ controls the oscillation period defined with a good accuracy by (25) with $\tau$ defined by (21) (where $L_{r}^{\text {eff,i }}$ value should be used for $L_{\mathrm{r}}$ ). $\delta_{\text {If }}$ has the strongest impact on the amplitude of the oscillations. The increase of $\delta_{\text {If }}$ leads to the decrease of the heat transfer coefficients which decreases the amplitude. In addition it leads to the decrease of the rate of change of $L_{\text {lf }}$ through (26) which decreases the amplitude even stronger. $\gamma$ has been introduced to decouple these two effects.

\section{Comparison and discussion}

The oscillation amplitude in the film evaporation/condensation model is large. In agreement with the above experimental observations, the meniscus penetrates both into condenser and evaporator during each oscillation. One can deduce that the superheated vapor model is inadequate; the evaporation/condensation model should be used instead.

One of the most important features of a PHP model is the existence of the instability threshold. Fig. 6a corresponds to the conditions just above the threshold. It is sufficient to decrease $T_{\mathrm{e}}$ or increase $T_{\mathrm{c}}$ by $1 \mathrm{~K}$ to prevent the oscillations. The behavior of the system under threshold (i.e. at stability) resembles that of Fig. 5a.

The modeled behavior of the liquid film in the evaporator corresponds to the experiment [20]. It is slowly evaporated during the

Table 1b

Values of adjustable parameters used to obtain Fig. $6 \mathrm{a}$ and b.

\begin{tabular}{lccl}
\hline Quantity & Fig. 6a & Fig. 6b & Units \\
\hline$(b)$ & & & \\
$\gamma$ & 0.47 & 0.47 & - \\
$\delta_{\text {If }}$ & 100 & 50 & $\mu \mathrm{m}$ \\
$L_{\mathrm{m}}$ & 10 & 10 & $\mu \mathrm{m}$ \\
$L_{\mathrm{r}}^{\text {eff, }}$ & 4 & 0.7 & $\mathrm{~m}$ \\
$L_{\mathrm{r}}^{\text {eff, }}$ & 1.7 & 2.1 & $\mathrm{~m}$ \\
\hline
\end{tabular}

meniscus receding into the condenser and may disappear completely when the meniscus attains the edge $\left(x=x_{\mathrm{f}}\right)$ during a short time period. The rate of the film evaporation increases with $T_{\mathrm{e}}$; at large $T_{\mathrm{e}}$ the film can be completely evaporated before the meniscus comes back into the evaporator.

A comparison of Fig. 6a and b with Fig. 2a and b shows a good qualitative agreement. The amplitude of oscillations for the low temperature case (a), which is close to the instability threshold, is smaller than for the high temperature case (b). Both experiments and simulations show less regular oscillations in the case (a) and more regular oscillations in the case (b). Some finer features of the behavior are also reproduced. In particular, these are the intermittency of the pressure minima in the case (a) or sharp maxima of the pressure in the case (b). Both in the theory and experiment, the pressure curve is steeper at expansion than at compression. This is probably because, at high amplitude oscillations, liquid film enters deeply into the evaporator and more liquid evaporates giving a large pressure rise in short time. While the theoretical and experimental frequencies coincide for each of two cases, the amplitudes correspond within about $15 \%$. However, it is not possible to achieve a complete coincidence of the amplitudes of both displacement and pressure by fitting the parameters of Table $1 \mathrm{~b}$. For equal $x$ amplitudes, the simulated $p_{\mathrm{v}}$ amplitude turns out to be larger than the experimental value. We attribute this discrepancy primarily to the deficiency of the viscous friction model discussed above which manifests itself also in the unrealistically large values of $L_{\mathrm{r}}^{\text {eff,f }}$ in Table $1 \mathrm{~b}$.

\section{Conclusions}

In the present paper we analyze the functioning of the PHP that consists of a single bubble and a single liquid plug. Such a system can develop an instability that leads to the liquid-meniscus oscillations. A corresponding experiment is performed. The meniscus displacement in the transparent condenser and the pressure variation are analyzed both close to the instability threshold and far from it. Close to the instability threshold the oscillations are less regular and have a smaller amplitude than far from it. In an attempt to better understand the existing PHP models $[13,11,12]$, we analyze their simplified version ("superheated vapor" model). Such a simplification allows some important parameters (in particular, the oscillation frequency) to be obtained analytically. We show that the existing models describe only small amplitude oscillations during which the meniscus stays in the condenser section. We propose a more coherent ("evaporation/condensation") model that allows the large amplitude oscillations (as observed experimentally) to be explained. The results of the latter model are compared with the experiment. The model succeeds in the description of almost all observable qualitative features of the oscillations. In particular, a smaller amplitude of each second oscillation near the oscillation threshold is reproduced. A good quantitative agreement is achieved. This model is thus suitable for the future modelling of the multi-bubble PHP.

\section{Acknowledgements}

Financial help for the academic visit of S. Khandekar to CETHIL during the summer of 2008 from INSA-Lyon is gratefully acknowledged. This author also received partial research funding from Indian Space Research Organization under sponsored Project No. ISRO/ME/20050083.

\section{References}

[1] H. Akachi, Structure of Micro-Heat Pipe, US Patent 5219020, 15 June 1993. 
[2] L.L. Vasiliev, Heat pipes in modern heat exchangers, Appl. Therm. Eng. 25 (1) (2005) 1-19.

[3] Y. Zhang, A. Faghri, Advances and unsolved issues in pulsating heat pipes, Heat Transfer Eng. 29 (1) (2008) 20-44.

[4] B.Y. Tong, T.N. Wong, K.T. Ooi, Closed-loop pulsating heat pipe, Appl. Therm. Eng. 21 (18) (2001) 1845-1862.

[5] Y. Miyazaki, M. Arikawa, Oscillatory flow in the oscillating heat pipe, in: Proceeding of the 11th International Heat Pipe Conference, Tokyo, Japan, 1999, pp. 143-148.

[6] J. Xu, Y. Li, T. Wong, High speed flow visualization of a closed loop pulsating heat pipe, Int. J. Heat Mass Transfer 48 (16) (2005) 3338-3351.

[7] K. Gi, F. Sato, S. Maezawa, High speed flow visualization of a closed loop pulsating heat pipe, in: Proceeding of the 11th International Heat Pipe Conference, Tokyo, Japan, 1999, pp. 149-153.

[8] P. Charoensawan, S. Khandekar, M. Groll, P. Terdtoon, Closed loop pulsating heat pipes: Part A: parametric experimental investigations, Appl. Therm. Eng. 23 (16) (2003) 2009-2020.

[9] H. Yang, S. Khandekar, M. Groll, Operational limit of closed loop pulsating heat pipes, Appl. Therm. Eng. 28 (1) (2008) 49-59.

[10] Y. Zhang, A. Faghri, M.B. Shafii, Analysis of liquid-vapor pulsating flow in a $U$-shaped miniature tube, Int. J. Heat Mass Transfer 45 (12) (2002) 25012508.
[11] R.T. Dobson, Theoretical and experimental modelling of an open oscillatory heat pipe including gravity, Int. J. Therm. Sci. 43 (2) (2004) 113-119.

[12] R.T. Dobson, An open oscillatory heat pipe water pump, Appl. Therm. Eng. 25 (4) (2005) 603-621.

[13] M.B. Shafii, A. Faghri, Y. Zhang, Thermal modeling of unlooped and looped pulsating heat pipes, J. Heat Transfer 123 (6) (2001) 1159-1172.

[14] P. Sakulchangsatjatai, P. Terdtoon, T. Wongratanaphisan, P. Kamonpet, M. Murakami, Operation modeling of closed-end and closed-loop oscillating heat pipes at normal operating condition, Appl. Therm. Eng. 24 (7) (2004) 9951008.

[15] van P. Carey, Liquid-Vapor Phase Change Phenomena, Hemisphere, Washington, DC, 1992

[16] Y. Zhang, A. Faghri, Heat transfer in a pulsating heat pipe with open end, Int. J. Heat Mass Transfer 45 (4) (2002) 755-764.

[17] M. Tabor, Chaos and Integrability in Nonlinear Dynamics: An Introduction, first ed., Wiley-Interscience, 1989.

[18] P.-G. de Gennes, Wetting: statics and dynamics, Rev. Mod. Phys. 57 (1985) 827-863.

[19] NIST Chemistry Webbook, NIST Standard Reference Database 69. <http:// webbook.nist.gov>.

[20] G. Lagubeau, Propulsion Par Moteur Pop-Pop, Master's Thesis, Supervisor: D. Quéré, PMMH-ESPCI, Paris, 2006. 